\title{
Transformer Winding Parameter Identification based on Frequency Response Analysis using Hybrid Wavelet Transform (WT) and Simulated Annealing Algorithm (SA) and Compare with Genetic Algorithm (GA)
}

\author{
Sajad Bagheri', Reza Effatnejad ${ }^{2 *}$ and Abolfazl Salami ${ }^{3}$ \\ 'Department of Electrical Engineering, Science and Research Branch, Islamic Azad University, \\ Arak-Iran; bagheri_ieee@yahoo.com \\ ²Electrical Engineering Department, Karaj Branch, Islamic Azad University, Karaj, Iran; Reza.efatnejad@kiau.ac.ir \\ ${ }^{3}$ Faculty of Electrical Engineering, ArakTechnology University, Arak, Iran; a_salami@iust.ac.ir
}

\begin{abstract}
Frequency response analysis method is used for certain evaluating and monitoring of the winding displacement and deformation of transformer core to detect incipient faults. Frequency response analysis is based on the graphical analysis and comparison of the current measured transfer function with a base current. In this paper we use compound of haar wavelet transform and intelligent Simulated Anealing Algorithms (SA) and finally we compare the achieved parameters with Genetic Algorithm (GA).
\end{abstract}

Keywords: Frequency Response Analysis, Haar Wavelet Transform, Genetic Algorithm, Simulated Annealing, Transformers Winding Parameter Identification, Traveling Wave Model

\section{Introduction}

Frequency Response Analysis (FRA) has been utilized as an off-line diagnosis test since last decade to investigate mechanical integrity of transformer. One of the most important issues raised in connection with any electrical equipment is to have an equivalent electrical model for that equipment to identify and analyze its transient and permanent behavior. Therefore, the identification and analysis of transformers performance the same as any other system require the availability of an electrical model of $i^{1,2}$. There are different models for different applications of the transformer. Since in the frequency response analysis method, frequency behavior of the transformer is studied in a wide range of frequency, having a model so that it can help to analyze the frequency behavior of the transformer or to use for sensitivity analysis applications is of great importance. FRA is an offline test ${ }^{3,4}$. However, a model that includes transformer characteristics over a wide frequency range has not been provided yet properly. In fact, due to the complexity and unknown behavior of high frequency coils, its modeling is complex 5 .

In the high-frequency modeling of transformer coils, two types of models are available to obtain the transfer function of the transformer, Traveling Wave Model and Detailed Model. In the Detailed Model, to study and calculate the voltage in points inside coils is regarded as suitable purposes of modeling. This method is used to study the transient behavior of coils. However, it is so obvious that a model of transformer coils is a complicated network of electric elements and includes some resistors, capacitors and inductors. How to consider the 
arrangement of elements and to analyze them is the first step for presenting a proper model ${ }^{1,7}$.

\section{Mathematical Model of Transformer Winding ${ }^{8-10}$}

In order to accurately model the transient behavior of transformer windings, we need models with a large number of nodes and parameters. This paper proposes a model that has fewer nodes and can accurately predict the behavior of a transformer in a wide range of frequencies. Significant studies have concentrated on wave propagation in transformer winding. The proposed method is based on wave travelling model.

To reach this level of conception, equivalent detail circuit of transformer winding has been considered as a model and demonstrated in Figures 1, 2.

According to Figure 2, the charging currents for infinitesimal length of transformer winding are given as equation (1).

$$
i_{c_{s}}=c_{s} \frac{\partial^{2} e}{\partial x \partial t}, i_{g}=g \frac{\partial e}{\partial x}
$$

where, $i_{c_{s}}$ is the internal capacitive current per unit conductor length, $c_{s}$ denotes series capacitance, $e$ is the potential to ground (time domain), $i_{g}$ represents external conductance current per unit conductor length and $g$ is the shunt turn to turn conductance. Also, discharging currents through shunt capacitance as well as conductance for infinitesimal length of transformer winding flow to ground are calculated as equation (2).

$$
i_{c_{g}}=c_{g} \frac{\partial e}{\partial t}, i_{G}=e G
$$

where, $i_{c_{g}}$ is the external capacitive current per unit conductor length, $c_{g}$ denotes shunt capacitance to ground, $i_{G}$ is the external conductance current per unit conductor length, and $G$ represents shunt conductance to ground. Equation (3) is valid if the transformer winding includes numerous number of turns and also if the detailed distribution of the wave propagation along a single turn length is not to be interested. Therefore, space derivative of current is calculated as equation (3),

$$
\frac{\partial}{\partial x}\left(-i+i_{c_{s}}+i_{g}\right)-i_{c_{g}}-i_{G}=0
$$

Where, $i$ is the conductor current. Substituting of equations (1) and (2) into equation (3) will lead to equation (4).

$$
\frac{\partial}{\partial x}\left(c_{s} \frac{\partial^{2} e}{\partial x \partial t}+g \frac{\partial e}{\partial x}-i\right)-c_{g} \frac{\partial e}{\partial t}-e G=0
$$

According to equation (4), space derivative of winding current for infinitesimal length of transformer winding is given as equation (5).

$$
\frac{\partial i}{\partial x}=c_{s} \frac{\partial^{3} e}{\partial x^{2} \partial t}+g \frac{\partial^{2} e}{\partial x^{2}}-c_{g} \frac{\partial e}{\partial t}-e G
$$

On the other hand, self-inductance of infinitesimal winding turn contributes an induced voltage. In addition, two adjacent turns coupled by the mutual inductance $\mu$ per unit length cause additional induced voltage in the turn. Therefore, space derivative of winding voltage considering conductor loss for infinitesimal length of transformer

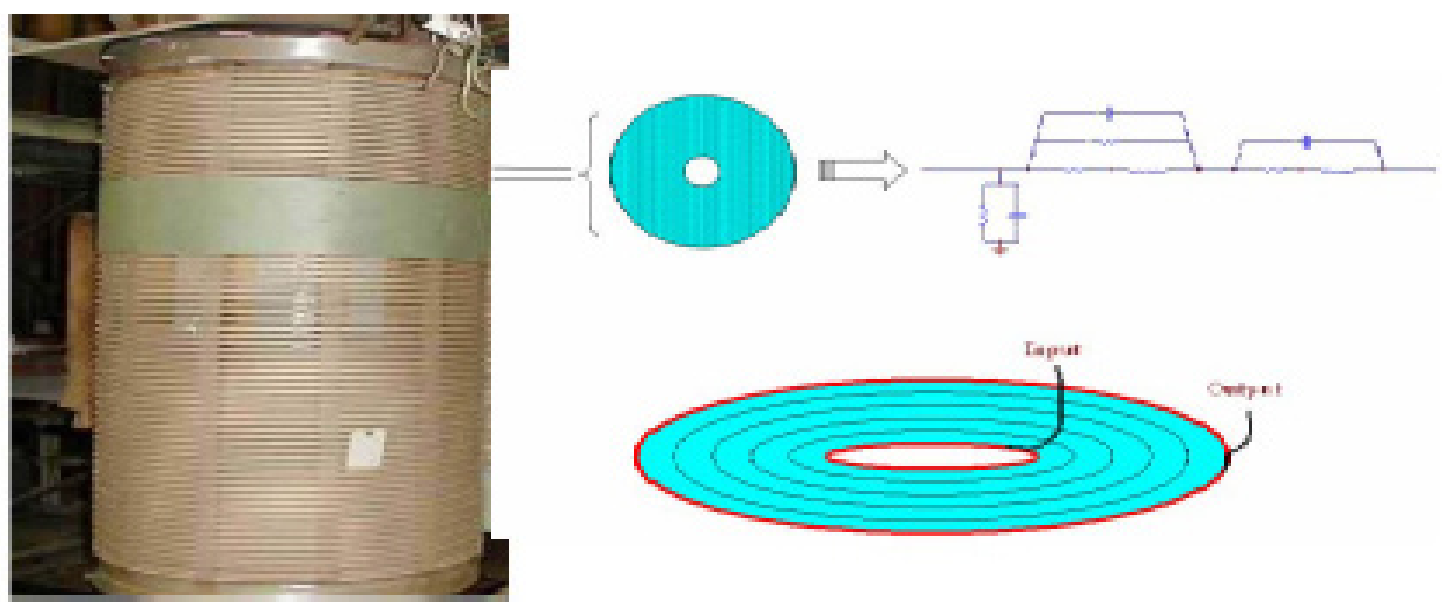

Figure 1. Continuous parameter model of transformer winding ${ }^{11}$. 
winding can be calculated as equation (6) while partial mutual inductances due to other conductors' turns are neglected.

$$
-\frac{\partial e}{\partial x}=\left(\gamma+\sum \mu\right) \frac{\partial i}{\partial t}+r i
$$

where,

$$
\left(\gamma+\sum \mu\right)=l
$$

$\gamma$ and $\mu$ are the self- and mutual-inductance, respectively, $r$ is the conductor resistance, and $l$ is the inductance coefficient. Subsequently, the voltage difference along the coil can be calculated as follow:

$$
\frac{\partial e}{\partial x}=-l \frac{\partial i}{\partial t}-r i
$$

In fact, the voltage as well as the current in a long coil varies from turn to turn as a function of time and also space. Current and voltage studies ${ }^{10}$ have been converted from time domain to frequency domain using Laplace transform while initial conditions have been supposed to be zero. Therefore, transferring equations (5) and (8) from time domain into frequency domain is given as equation (9).

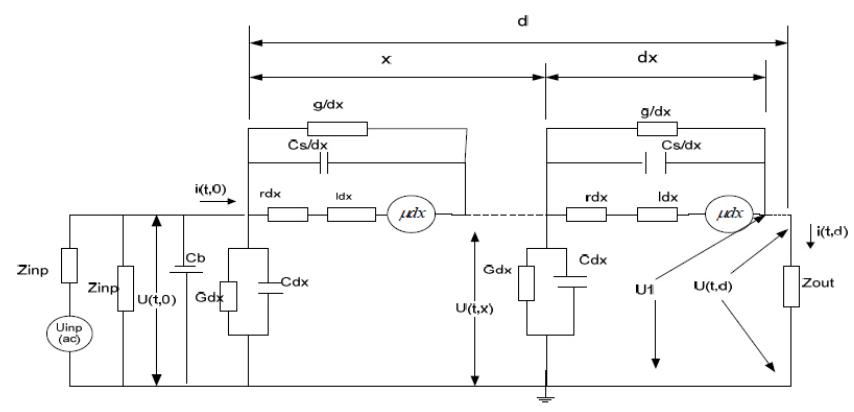

(a)

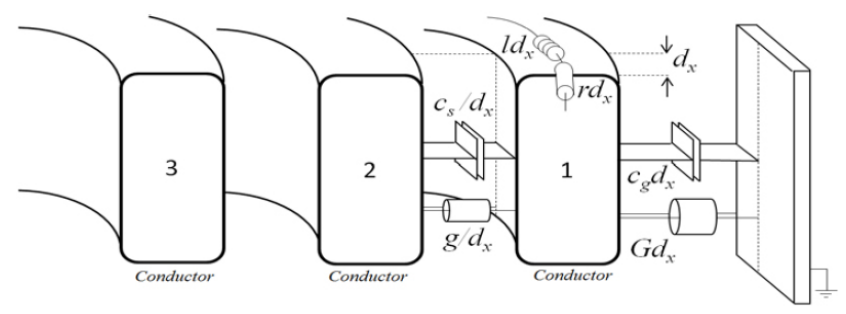

(b)

Figure 2. Equivalent detail circuit of a transformer winding ( $\mathrm{dx}$ is the infinitesimal length of winding), (a) Entire winding schematic, (b) Close view ${ }^{10,11}$.

$$
\begin{aligned}
& \frac{\partial}{\partial x}\left[\begin{array}{l}
v(j \omega, x) \\
I(j \omega, x)
\end{array}\right]=-\left[\begin{array}{cc}
j \omega l+r & 0 \\
0 & j \omega c_{g}+G
\end{array}\right]\left[\begin{array}{l}
I(j \omega, x) \\
v(j \omega, x)
\end{array}\right]+ \\
& {\left[\begin{array}{cc}
0 & 0 \\
0 & \left(j \omega c_{s}+g\right)
\end{array}\right] \frac{\partial^{2}}{\partial x^{2}}\left[\begin{array}{l}
I(j \omega, x) \\
V(j \omega, x)
\end{array}\right]}
\end{aligned}
$$

where, $v$ represents potential to ground (frequency domain), $I$ is the conductor current (frequency domain), $\omega$ is the angular frequency, and $j$ denotes imaginary operator. According to equation (9), differential equation for voltage propagation along transformer winding will be as follow:

$$
-\frac{\partial^{2} V(j \omega, x)}{(j \omega l+r) \partial x}=-\left(j \omega c_{g}+G\right) V(j \omega, x)+\left(j \omega c_{s}+g\right) \frac{\partial^{2} V(j \omega, x)}{\partial x^{2}}
$$

$$
-\frac{\partial^{2} V(j \omega, x)}{\partial x^{2}}-\frac{(j \omega l+r)\left(j \omega c_{g}+G\right)}{\left(1+(j \omega l+r)\left(j \omega c_{s}+g\right)\right.} v(j \omega, x)=0
$$

Therefore, the solution for the voltage at any point $x$ along the transformer winding is given as equation (12).

$$
\begin{aligned}
& V(j \omega, x)=A_{1} e^{-\beta x}+A_{2} e^{\beta x} \\
& \beta=\sqrt{\frac{(j \omega l+r)\left(j \omega c_{g}+G\right)}{1+(j \omega l+r)\left(j \omega c_{s}+g\right)}}
\end{aligned}
$$

$\beta$ denotes the propagation coefficient and $A_{1}$ and $A_{2}$ are constants that depend upon the boundary conditions. It is worth noting that $x=0$ and $x=\lambda$ are assumed as the neutral- and line terminal points of the transformer winding, respectively where the winding length is $\lambda$. According to equation (9), the current at any point of the transformer winding is calculated as equation (14).

$$
I(j \omega, x)=\frac{\beta}{j \omega l+r}\left(A_{1} e^{-\beta x}-A_{2} e^{\beta x}\right)
$$

On the other hand, logarithmic scale of frequency response magnitude for a transformer winding is calculated as equation (15). Also, Figures 3, 4 demonstrates common FRA test setups for a single winding and also transformer including the source voltage as well as $V_{\text {in }}$ and $V_{\text {out }}$. Based on equation (15) and Figures 3, 4, the output voltage respect to input voltage should be calculated to reach frequency response.

$$
K=20 \log _{10}\left(\frac{V_{\text {out }}}{V_{\text {in }}}\right)
$$

where, $\mathrm{K}$ is the frequency response magnitude. 


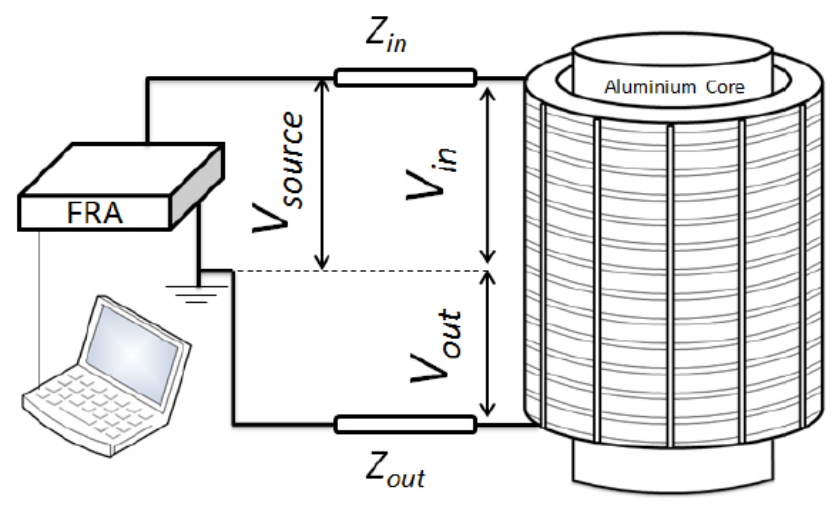

Figure 3. Common FRA test setup, for single winding ${ }^{10}$.

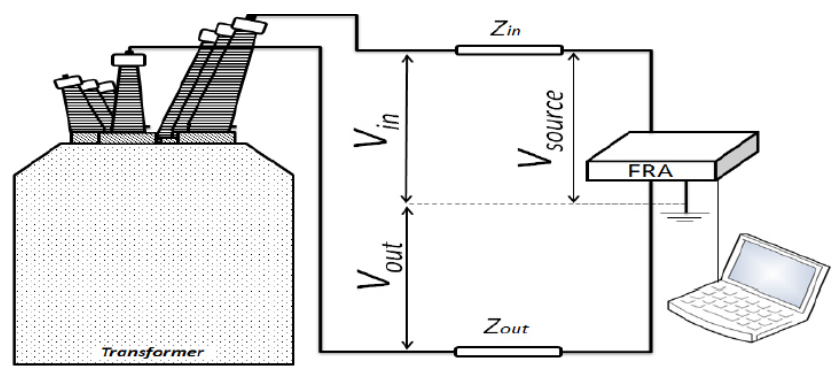

Figure 4. Common FRA test setup, For Transformer ${ }^{10}$.

According to equation (15) and the FRA test setup shown in Figures 3, 4; $V_{\text {in }}$ is measured at the line-terminal of the winding while $V_{\text {out }}$ is measured at the neutralterminal. Also, $Z_{\text {in }}$ and $Z_{\text {out }}$ are the input and output measurement cable impedances and commonly represented by $50 \Omega$ resistors. Since, $Z_{\text {out }}$ has small value compared to equivalent impedance of the winding, it is approximated that $V_{\text {out }} \leq V_{\text {in }}$. Therefore in equation (12), $A_{1}=-A_{2}$ and frequency response trace can be calculated as equation (16).

$$
\frac{V_{\text {out }}}{V_{\text {in }}}=\frac{Z_{\text {out }} I(j \omega, 0)}{V(j \omega, \lambda)}=\frac{Z_{\text {out }} \beta}{j \omega l+r}\left(\frac{e^{-\beta 0}+e^{\beta 0}}{e^{-\beta \lambda}-e^{\beta \lambda}}\right)
$$

It should be emphasized that according to equation (16) and above mentioned assumption, transformer frequency response magnitude exhibits small value especially in very high frequencies. Although, according to equation (15) when equation (16) is subject to logarithm operation, reported frequency response magnitude in Bode diagram would be significant and completely traceable. Simplification of (16) using complex algebra is given as equation (17).

$$
\begin{aligned}
& \frac{V_{\text {out }}}{V_{\text {in }}}=\xi\left(\frac{1}{j \sin (j \beta \lambda)}\right) \\
& Z_{\text {out }} \beta(j \omega l+r)^{-1}=\xi, \quad \lambda=N_{w} d
\end{aligned}
$$

where, $N_{w}$ is the number of winding turns, and $d$ is the average length of a conductor turn.

Transfer function resonances occur when the denominator in equation (17) experiences minimum absolute value. It can be yielded once $\beta^{2} \lambda^{2}=-(k \Pi)^{2}$. Therefore,

$$
\begin{aligned}
& \left(\frac{(j \omega l+r)\left(j \omega c_{g}+G\right)}{1+(j \omega l+r)\left(j \omega c_{s}+g\right)}\right) \lambda^{2}=-k^{2} \pi^{2} \\
& k=1,2,3, \ldots \ldots \ldots \ldots \ldots . . .
\end{aligned}
$$

Then,

$$
\begin{aligned}
& \omega^{2}\left(l_{c_{g}}+k^{2} \pi^{2}\left(\lambda^{2}\right)^{-1} l_{c_{s}}\right)- \\
& \omega\left(\left(r_{c_{g}}+l_{G}\right) j+k^{2} \pi^{2}\left(\lambda^{2}\right)^{-1}\left(r_{c_{s}}+l_{\mathrm{g}}\right) j\right)- \\
& r_{G}-k^{2} \pi^{2}\left(\lambda^{2}\right)^{-1} r_{g}=k^{2} \pi^{2}\left(\lambda^{2}\right)^{-1}
\end{aligned}
$$

Moving from low frequencies to mid and ultimately high frequencies will lead to $\omega^{2} \succ \succ \succ \omega$, therefore,

$$
\omega^{2}\left(l_{c_{g}}+k^{2} \pi^{2}\left(\lambda^{2}\right)^{-1} l_{c_{s}}\right)=k^{2} \pi^{2}\left(\lambda^{2}\right)^{-1}
$$

Hence, resonance frequency $(f r)$ will be calculated as (21).

$$
f_{r}=\frac{k}{2 \lambda}\left(\frac{1}{\sqrt{\left.c_{g}+c_{s} k^{2} \pi^{2}\left(\lambda^{2}\right)^{-1}\right) l}}\right)
$$

\section{Simulated Annealing Algorithm}

This algorithm was discovered in 1980. This method, because of its simplicity and high efficiency in solving optimization problems, has a special place among the techniques used for search and exploration.

Cooling the particles mildly causes the particles to have the opportunity to minimize their energy level and the next time they have the appropriate arrangement. The slow process of cooling to achieve excellent properties is called Anneal or gradual freezing. If a solid material heated up to the melting point and then cooled until solid, cooled solid structural properties will depend on its cooling rate. Gradual freezing also is modeled from the annealing algorithm, and it use temperature control algorithm to improve the solution. 
Gradual freezing method simulates the change in energy when the system reaches a steady state of frozen. Simulated Annealing algorithm (SA) is a random method that uses statistical mechanism to achieve the optimal solution.

SA algorithm acts on the two principles of statistical physics:

A: Thermodynamic equilibrium with Boltzmann's factor.

A1: If this rule is that when a specified temperature reaches thermodynamic equilibrium, the possibility of a physical system having energy will be proportional to Boltzmann factor.

A2: Under this rule, the probability of accepting solutions at different temperatures is not similar.

B: Thermodynamic equilibrium with the Metropolis algorithm.

B1: This rule shows how to achieve thermodynamic equilibrium at a specific temperature.

B2: A limited series of basic changes is considered. If changing the form leads to a decrease in the objective function or power, it will be accepted. Otherwise a random number between 0 and 1 is generated .If the number is less than the value of the function, changes will be accepted.

$$
P(\Delta E)=E^{-\Delta E / K T}
$$

\subsection{The Steps of the Algorithm SA}

A: The initial solution: creating an initial solution $\mathrm{x} 1$ in the $\mathrm{X}$ set.

B: Markov chain formation: Replication of neighboring solutions of the current solution until the Markov chain ends.

C: Temperature reduction: reduce temperature to the specified value.

D: Stop: if the stopping rule is satisfied, stop .Otherwise, go to the $\mathrm{B}$ stage.

Now, according to the description given above and the references ${ }^{12-15}$, we can easily optimize the parameters of transformer winding with Simulated Annealing algorithms (SA) by using Matlab software, and compare it with other smart methods (Table 1).

\section{Genetic Algorithm}

Genetic Algorithm is a Biological evolution based on learning procedure. Biological evolution is a genetic
Table 1. SA Parameters

\begin{tabular}{ll}
\hline Parameter & \multicolumn{1}{c}{ Value } \\
\hline Initial temperature & 100 \\
Final temperature & $9.04626 \mathrm{e}-007$ \\
Consecutive rejections & 12248 \\
Number of function calls & 248012 \\
\hline
\end{tabular}

algorithm based on learning procedure. This method was introduced in 1970 by John Holland that is also called Evolutionary Algorithms.

The applications of genetic algorithms can be noted as follows:

Optimization, Automatic programming, Machine learning, Economics, Operations research, Ecology, Studies of evolution and Learning, and Social systems.

Genetic Algorithm because of high convergence speed, high accuracy and high efficiency can be used in this paper to optimize the parameters of transformer winding.

\section{1 Implementation Methodology}

A simple GA procedure is shown in Figure $5^{16}$ :

a) Choose the first population of individuals;

b) Evaluate the fitness of each individual in that population;

c) Select the best-fit individuals for reproduction;

d) Breed new individuals through crossover and mutation operations to give birth to offspring's;

e) Evaluate the individual fitness of each of the new individuals;

f) Replace the least-fit population with the new individuals.

The general flowchart of Genetic algorithm is shown as follows:

In this paper, to optimize the parameters of transformer winding, the genetic toolbox of the Matlab with the parameters given in Table 2, is more used.

\section{Simulation Result and Comparison the Results of GA, SA Algorithms}

First obtain transformer reference transfer function according to Figures 6,7 , and then it is resolved using 


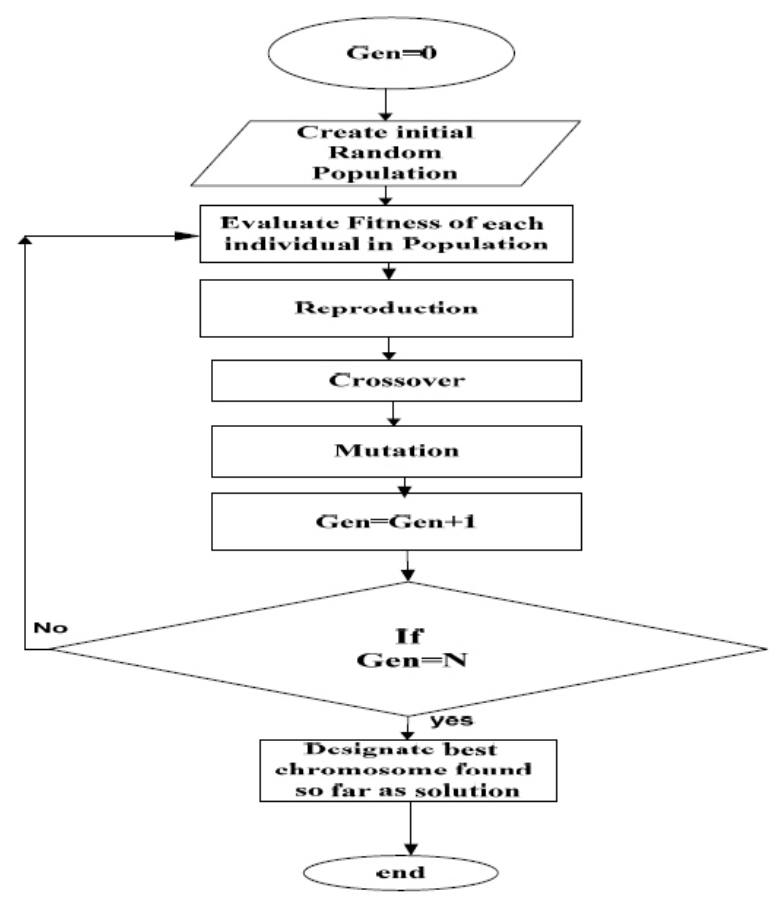

Figure 5. Flow chart describing the GA.

Table 2. GA Parameters

\begin{tabular}{ll}
\hline Parameter & \multicolumn{1}{c}{ Value } \\
\hline Selection Function & Stochastic Uniform \\
Population Type & Double Vector \\
Scaling Function & Rank \\
Population Size & 50 \\
Generation & 500 \\
Time Limit & 200 \\
Stall Time Limit & 100 \\
\hline
\end{tabular}

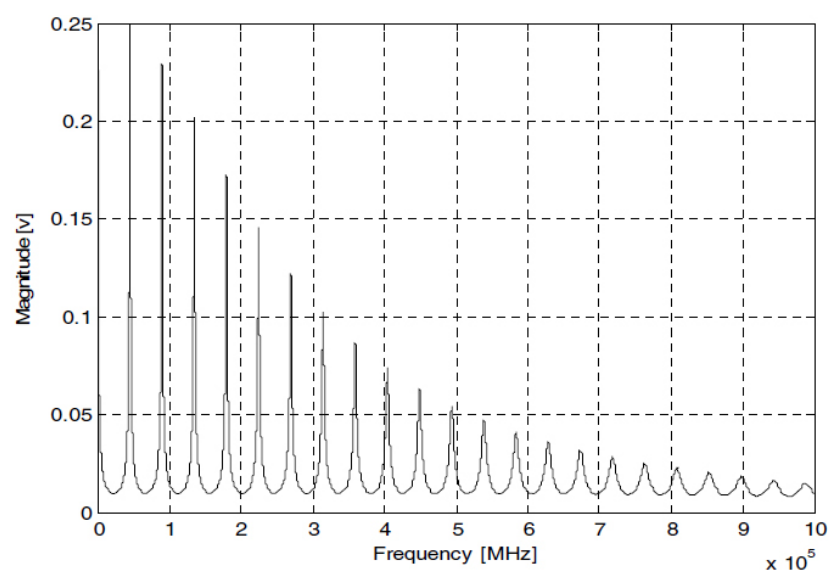

Figure 6. The reference transfer functions of transformer winding. haar wavelet transform through passing low-pass filter and calculating approximate signal and passing high-pass filter and calculating details signal according to Figure 8. After determining the best frequency by Wavelet Haar, it's the smart way for SA, GA to apply the optimal frequency parameters of transformer winding in this area. After obtaining the results, it is shown in Table 3 and is compared with reference values obtained from the laboratory.

Finally, by putting the parameters of the transfer function obtained from the mathematical model of transformer winding, which are explained in part 2, the frequency response phase versus degree and frequency response magnitude, depending on the voltage, are shown in Figures 10 and 11. Also, the described flowchart of this method has been presented in Figure 9.

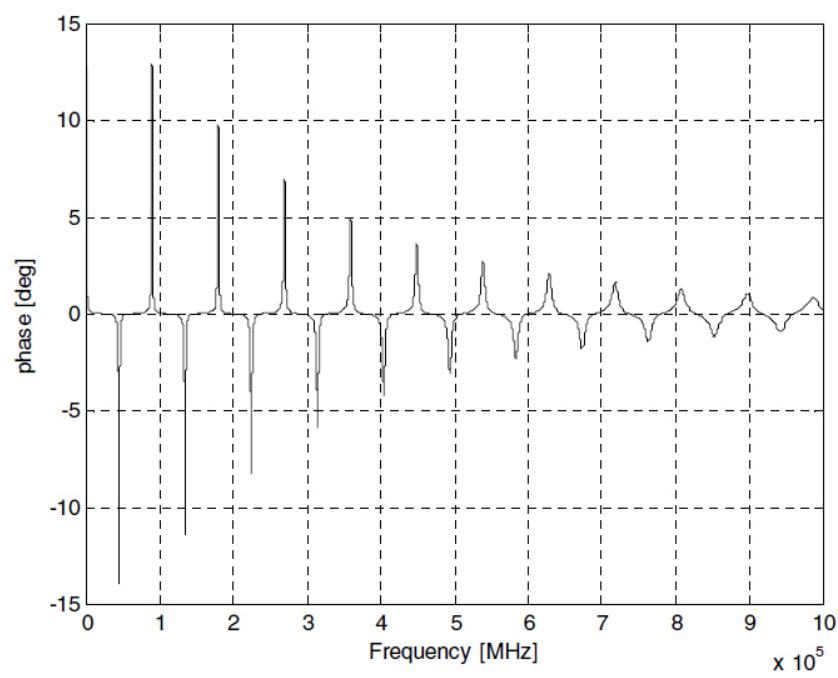

Figure 7. The reference transfer function phase of transformer winding.
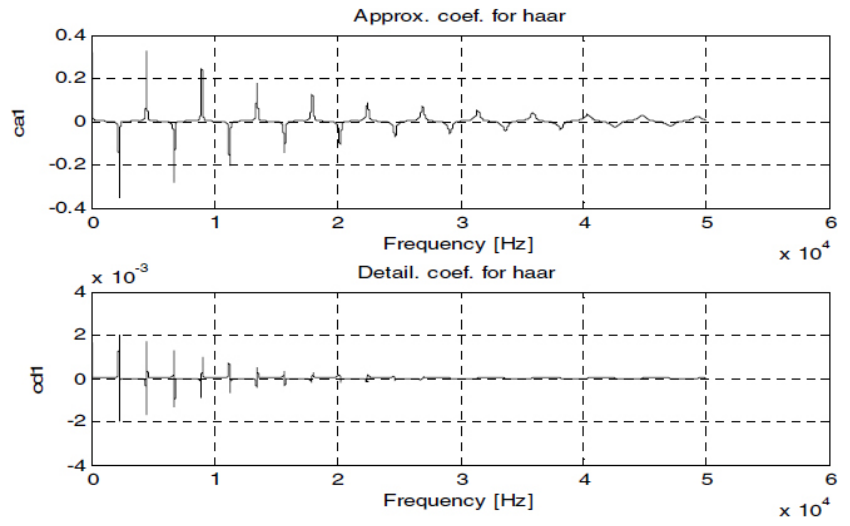

Figure 8. The magnitudes of resolved frequency transfer function of Figure 6 using haar wavelet transform; Approximate coefficient, Detail coefficient. 
The haar function has got a pair of rectangular pulse, and is the simple stand oldest narrow basic wavelet.

Table 4 summarizes the reference parameter and the identified parameter values with SA and GA.

Table 3. Transformer Parameters

\begin{tabular}{lcc}
\hline Parameter & symbol & value \\
\hline Transformer Power (MVA) & $\mathrm{S}$ & 30 \\
Transformer ratio (KV) & $\mathrm{N} 2 / \mathrm{N} 1$ & $63 / 20$ \\
Vector Group & - & YNd11 \\
Number of disks & $N_{d}$ & 46 \\
Number of turns per a disk & $N_{t}$ & 6 \\
Self-inductance, $\mu \mathrm{H} / \mathrm{m}$ & $\mathrm{L}$ & 6.3291 \\
Resistance W/m & $\mathrm{r}$ & 0.01287 \\
Ground capacitance, $\mathrm{pF} / \mathrm{m}$ & $\mathrm{C}$ & 1.01634 \\
Inter turn capacitance, $\mathrm{pF} / \mathrm{m}$ & $C_{s}$ & 0.0982 \\
Bushing capacitance, $\mathrm{pF} / \mathrm{m}$ & $C_{b}$ & 450 \\
Ground conductivity, $\mathrm{nSi} / \mathrm{m}$ & $\mathrm{G}$ & 0.500 \\
Inter turn conductivity, $\mu \mathrm{Si} / \mathrm{m}$ & $\mathrm{g}$ & 40.102 \\
Average length of turn, $\mathrm{m}$ & $\mathrm{a}$ & 2.576 \\
Total length of winding, $\mathrm{m}$ & $\mathrm{d}$ & 2758 \\
\hline
\end{tabular}

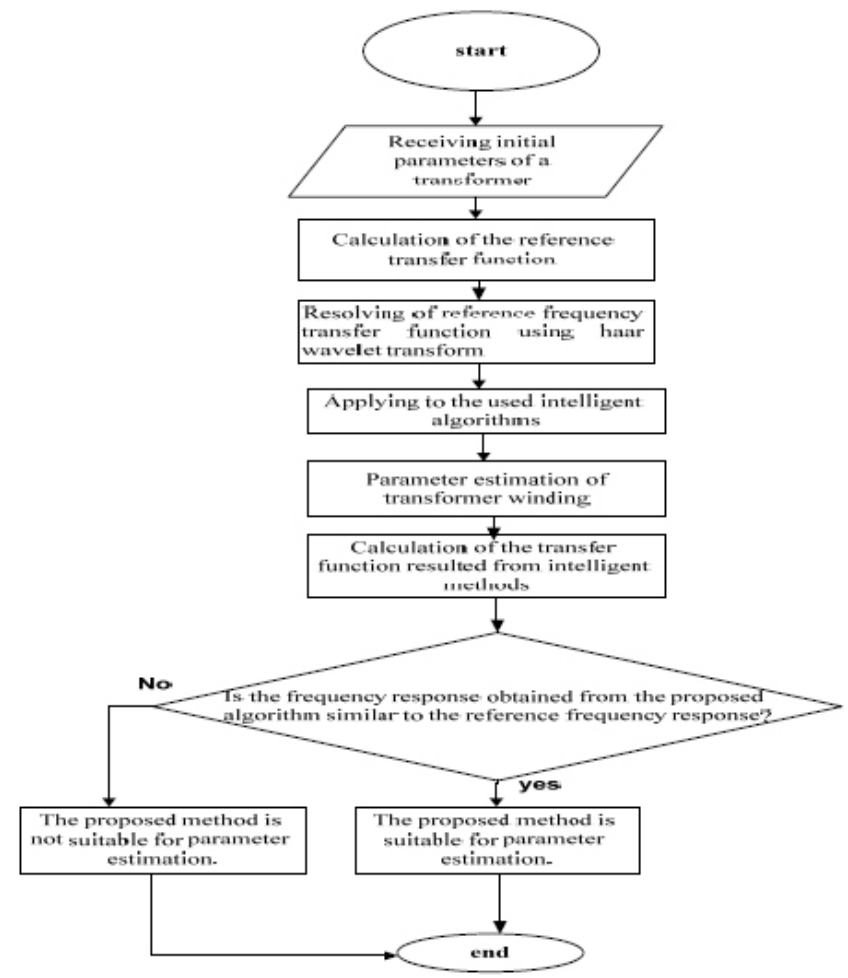

Figure 9. Flow chart describing the methodology used in the analysis ${ }^{11}$.

\section{Conclusion}

The frequency response analysis method is based on changes in physical structure and winding isolation, and traveling wave model is an appropriate model for further consideration and identification of the method.

The traveling wave model compared with models based on equivalent circuits of intensive parameters (detailed model) has the inherent simplicity that can be useful in designing a new system of continuous control and care of transformer status.

In this paper, first we obtained the transformer reference frequency response using the traveling wave model and separated it with the function of wavelet to determine the appropriate frequency range and apply to intelligent

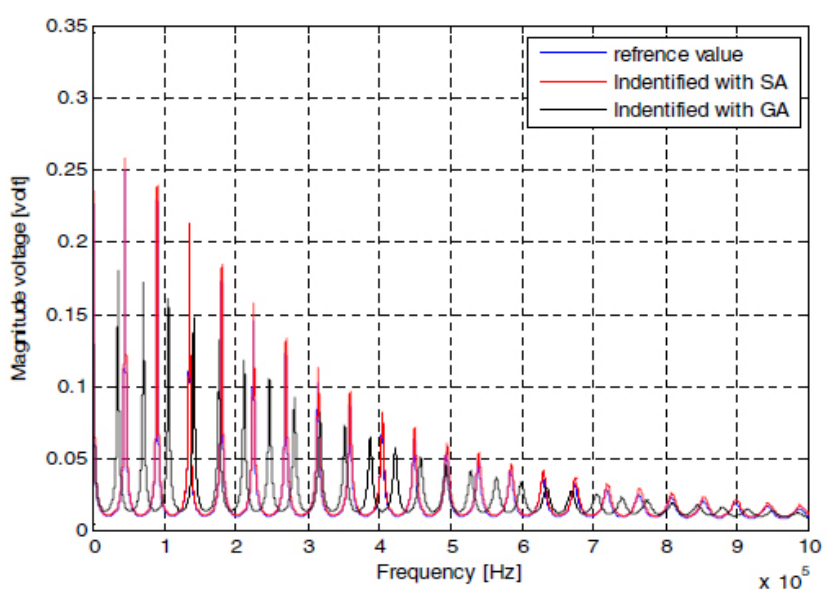

Figure 10. Comparison of the transfer function magnitude frequency response: indentified with GA, SA and reference.

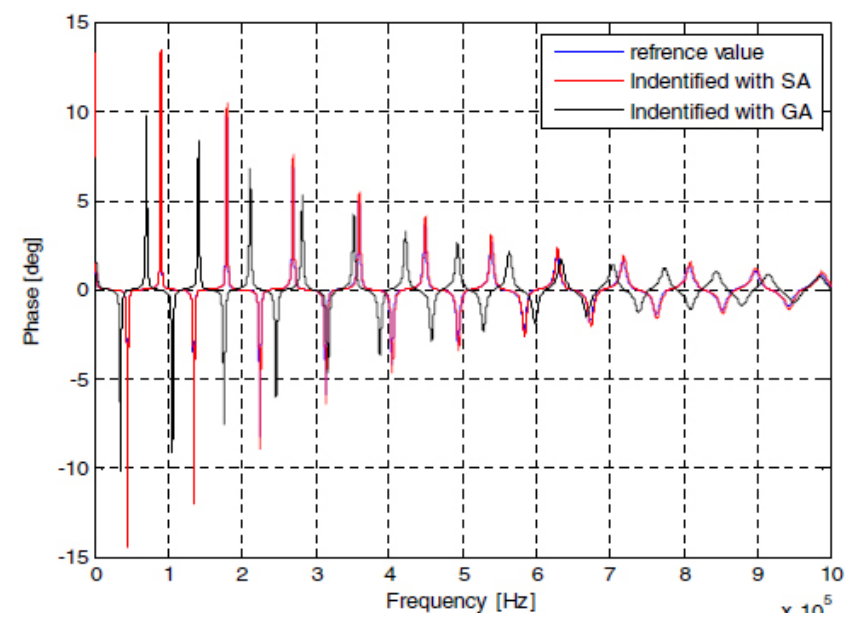

Figure 11. Comparison of the transfer function phase's frequency response: indentified with GA, SA and reference. 
Table 4. Comparison of the reference and identified parameters of the transformer winding model.

\begin{tabular}{|c|c|c|c|c|c|}
\hline \multirow[t]{2}{*}{ Parameter } & \multirow[t]{2}{*}{ Reference Value } & \multicolumn{2}{|c|}{ Indentified Value } & \multicolumn{2}{|c|}{$\begin{array}{l}\text { Deviation from } \\
\text { the reference }\end{array}$} \\
\hline & & SA & GA & SA & GA \\
\hline $\mathrm{L}$ & 6.3291 & 5.8885 & 5.4281 & 0.4406 & 0.9010 \\
\hline $\mathrm{r}$ & 0.01287 & 0.01131 & 0.0389 & 0.0015 & 0.0260 \\
\hline $\mathrm{C}$ & 1.01634 & 1.0896 & 1.5832 & 0.07326 & 0.5668 \\
\hline $\mathrm{C}_{\mathrm{s}}$ & 0.0982 & 0.0667 & 0.2510 & 0.0315 & 0.1528 \\
\hline $\mathrm{C}_{\mathrm{b}}^{\mathrm{s}}$ & 450 & 450 & 450 & 0 & 0 \\
\hline
\end{tabular}

methods. Then, the method of Simulated Annealing Algorithm is adopted to estimate the parameters of power transformer winding and the resultant transfer functions are compared with the Genetic Algorithm. Finally, of comparing the obtained Figures $(10,11)$, we found out that the exact diagnosis of parameters in the SA method is stronger than that of the GA method.

\section{References}

1. Shintemirov A, Tang W H et al. Transformer core parameter identification using frequency response analysis. IEEE Trans Magn. 2010; 46 (1):141-49.

2. Mukherjee P, Satish L. Construction of equivalent circuit of a single and isolated transformer winding from FRA data using the ABC algorithm. IEEE Trans. on power Del. 2012; 27(2):963-70.

3. Hashemnia N, Abu-Siada A et al. Characterization of transformer FRA signature under various winding faults. IEEE International Conference on Condition Monitoring and Diagnosis. 2012; 446-49.

4. Pourhossein K, Gharehpetian GB et al. A probabilistic feature to determine type and extent of winding mechanical defects in power transformers, Electric Power Systems Research. 2011; 82:1-10.

5. Ji TY, Tang WH et al. Detection of power transformer winding deformation and variation of measurement connections using a hybrid winding model. Elec Power Syst Res. 2012; 87:39-46.

6. Behjat V, Vahedi A et al. Sweep frequency response analysis for diagnosis of low level short circuit faults on the windings of power transformers: an experimental study. Electric Power and Energy System. 2012; 42:78-90.

7. Shintemirov A, Tang WJ et al. Improved modeling of power transformer winding using bacterial swarming algorithm and frequency response analysis. Elec Power Syst Res. 2010; 80:1111-20.

8. Bewley LV. Travelling waves on electric power systems, traveling waves on transmission systems. New York: John Wiley; 1942.

9. Bewley LV. Travelling waves on transmission systems, the interest of the advanced course in engineering of the general electric company. New York: John Wiley; 1933.

10. Bagheri M, Phung BT et al. Transformer frequency response analysis: mathematical and practical approach to interpret mid-frequency oscillations. IEEE Trans Power Deliv. 2013; 20(6):1962-70.

11. Bagheri S, Effatnejad R et al. Technical condition monitoring of power transformers based on frequency response analysis using hybrid Meyer Wavelet Transform (MWT) and Bacterial Swarm Algorithm (BSA) and Particle Swarm Optimization (PSO). International Journal on Technical and Physical Problems of Engineering. 2013; 5(17):161-68.

12. Fodorean D, Idoumghar L et al. Simulated annealing algorithm for the optimization of an electrical machine, IET Electric Power Applications. 2011; 6(9):735-42.

13. Wang Y, Li F et al. Reactive power planning based on fuzzy clustering, gray code, and simulated annealing. IEEE Trans Power Syst. 2011; 26(4):2246-55.

14. Xavier-de-Souza S, Suykens JA et al. Coupled simulated annealing. IEEE Transactions on Systems. 2010; 40(2):320-35.

15. Martins TC, de Camargo EDL et al. Image reconstruction using interval simulated annealing in electrical impedance tomography. IEEE Trans Biomed Eng. 2012; 59(7):1861-70.

16. Shabestary MM, Ghanizadeh AJ et al. Ladder network parameters determination considering nondominant resonances of the transformer winding. IEEE Trans Power Deliv. 2013; 1-10. 